Acta Crystallographica Section B

Structural Science, Crystal Engineering and Materials

ISSN 2052-5192

\author{
Alexander J. Blake ${ }^{\mathrm{a} *}$ and Marc de \\ Boissieu $^{\text {b* }}$
}

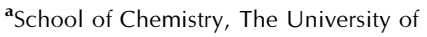
Nottingham, University Park, Nottingham NG7 2RD, England, and ' ${ }^{\mathbf{b}}$ Science de I'Ingénierie des Matériaux et Procédés (SIMaP), 1130 Rue de la Piscine, BP 75, 38402 Saint Martin d'Hères CEDEX, France

\title{
Expanding the scope and influence of Acta Crystallographica Section B
}

\section{Introduction}

Acta Crystallographica Section B celebrates its 45th birthday in 2013, having emerged from the separation of the single journal Acta Crystallographica into Sections $A$ and $B$ in 1968. A further split in 1983 yielded three journals, namely Section A: Foundations of Crystallography, Section B: Structural Science and Section C: Crystal Structure Communications. The possibilities for depth and breadth of structural description and discussion have been important features available to authors in Acta Crystallographica Section B. The journal publishes scientific articles related to the structural science of compounds and materials in the widest sense. Knowledge of the arrangements of atoms, including their temporal variations and dependencies on temperature, pressure and other conditions, is often the key to understanding physical and chemical phenomena and is crucial for the design of new compounds, materials and supramolecular devices. Acta Crystallographica Section $B$ is the forum for publication of contributions on these topics. The journal covers scientific developments based on experimental studies as well as those based on theoretical approaches, including crystal structure prediction, structure-property relationships and database analysis.

\section{Journal focus fit for the future}

The recent elaboration of the journal's subtitle to Structural Science, Crystal Engineering and Materials reflects its continued commitment to publishing structural science in all its varieties while highlighting crystal engineering and materials science as areas of particular interest for now and the future. The underlying ambition is to attract more of the excellent science we currently publish while extending into these new areas. Coinciding with the International Year of Crystallography (IYCr) in 2014, all IUCr journals including Acta Crystallographica Section B will undergo significant changes to maintain their attractiveness to broad communities of scientists, and to enhance their stature in the publishing world by expanding into, catering for and promoting exciting areas of developing science.

\section{Journal development}

The required development of Acta Crystallographica Section B is already underway, for example with the re-introduction of lead/feature articles and of special issues of the journal. The existing strategy will be extended and further elaborated through consultation, including with the journal's editorial team and relevant IUCr Commissions, but comments are also invited from current and potential future authors and readers, including on the plans outlined below. The journal will appoint new Co-editors as required, not only to maintain expertise in existing areas of activity but also to emphasize its commitment to attracting papers from the new areas identified below. The key challenge for the journal is to determine what will make it worth an author's time and effort preparing and submitting an article, and delivering it.

\section{Journal scope and keywords}

A new and expanded set of keywords has recently been drawn up to define the scope of Acta Crystallographica Section B and thereby guide and attract authors. This list is not fixed or exclusive and will be kept under review. Currently it comprises: aperiodic crystals, charge densities, chemical bonding, coherent scattering, crystal engineering, 
crystal growth and epitaxy, crystal-structure prediction, database analysis, diffuse scattering, electron crystallography, electron densities, electron, neutron and X-ray diffraction and scattering, functional materials, magnetic structures, mineralogy, NMR crystallography, phase transitions in materials, porous materials, powder diffraction, soft condensed matter, solid-state physics and chemistry, structural dynamics, structural materials, structural schematics, structures under extreme conditions, thin films and time-resolved studies. The identification of these keywords also serves to define the distinct and evolving identities of Section $B$ and Section $C$ : the plans for developing the latter are outlined in a recent editorial (Linden, 2013).

Below are some of the seminal papers that have appeared in the journal that also illustrate the breadth of its scope:

On rigid-body motion of molecules in crystals (Schomaker \& Trueblood, 1968)

Effective ionic radii in oxides and fluorides (Shannon \& Prewitt, 1969)

Graph-set analysis of hydrogen-bond patterns in organic-crystals (Etter et al., 1990)

Bond-valence parameters for solids (Brese \& O'Keeffe, 1991)

The Cambridge Structural Database: a quarter of a million crystal structures and rising (Allen, 2002)

Novel tools for vizualizing and exploring intermolecular interactions in molecular crystals (McKinnon et al., 2004)

Towards crystal structure prediction of complex organic compounds a report on the fifth blind test (Bardwell et al., 2011)

\section{Lead and feature articles}

The current plan for the journal's development includes the commissioning and publication of a regular series of timely and influential lead and feature articles: in 2013 we have already published 'The charge-flipping algorithm in crystallography' by Palatinus (2013), 'The generalized invariom database (GID)' by Dittrich et al. (2013) and 'Why don't we find more polymorphs?' by Price (2013), and further articles spanning the scope of the journal are planned. These will also help to define the expanded scope of the journal and attract high-quality papers from the featured and cognate fields of research.

\section{Special issues}

We foresee a similar but complementary role for our planned special issues of Acta Crystallographica Section B. We are very grateful to the Guest Editors who have already agreed to prepare the following special issues which are scheduled to appear over the next year:

Crystal Engineering edited by A. D. Bond (University of Copenhagen, Denmark)

Non-ambient Crystallography edited by D. G. Billing (University of the Witwatersrand, South Africa) and A. Katrusiak (Adam Mickiewicz University, Poznan, Poland)

Crystal Structure Prediction edited by G. M. Day (University of Southampton, UK) and C. H. Gørbitz (University of Olso, Norway)

\section{Outlook}

Taken together, the changes described above will ensure that we increase our influence by attracting broad science communities and their outstanding papers, elevating Acta Crystallographica Section $B$ to become a scientifically richer and more diverse journal which will demonstrate enhanced impact throughout its chosen fields. As with all IUCr journals, it should form a natural home for papers which emphasize high-quality science.

\section{References}

Allen, F. H. (2002). Acta Cryst. B58, 380-388.

Bardwell, D. A. et al. (2011). Acta Cryst. B67, 535-551.

Brese, N. E. \& O'Keeffe, M. (1991). Acta Cryst. B47, 192-197.

Dittrich, B., Hübschle, C. B., Pröpper, K., Dietrich, F., Stolper, T. \& Holstein, J. J. (2013). Acta Cryst. B69, 91-104.

Etter, M. C., MacDonald, J. C. \& Bernstein, J. (1990). Acta Cryst. B46, 256-262.

Linden, A. (2013). Acta Cryst. C69, 313-314.

McKinnon, J. J., Spackman, M. A. \& Mitchell, A. S. (2004). Acta Cryst. B60, 627-668.

Palatinus, L. (2013). Acta Cryst. B69, 1-16.

Price, S. L. (2013). Acta Cryst. B69, 313-328.

Schomaker, V. \& Trueblood, K. N. (1968). Acta Cryst. B24, 63-76.

Shannon, R. D. \& Prewitt, C. T. (1969). Acta Cryst. B25, 925-946. 